\title{
RENORMALIZATION GROUP APPROACH IN NEWTONIAN COSMOLOGY
}

\author{
Y. SOTA, T. KOBAYASHI AND K. MAEDA \\ Department of Physics, Waseda University \\ T. KUROKAWA AND M. MORIKAWA \\ Department of Physics, Ochanomizu University \\ AND \\ AKIKA NAKAMICHI \\ Gumma Astronomical Observatory
}

Since the real Universe is clumpy and inhomogeneous even at rather large scale such as $100 \mathrm{Mpc}$, we may wonder whether the isotropic and homogeneous Friedmann model is valid when we compare some cosmological parameters with the observed values. The observed Hubble parameter $H_{0}$ or the density parameter $\Omega_{0}$ might be deviated from the theoretically expected ones from the Friedmann model. In fact, the density parameter $\Omega_{0}$ seems to vary depending on the observational methods and the distance scales. Furthermore, our Universe seems to possess a fractal property in the galaxy distributions at least at scale up to $10 \mathrm{Mpc}$.

In order to investigate such a non-standard feature of the Universe, we apply a renormalization group (RG) method to the Newtonian dust universe whose averaged variables have a scaling property expected from a fractal distribution. The averaged Newtonian fluid equations for dust are invariant under a scaling transformation of the proper distance with a time shift. Pulling them back to the original time by the dynamical equations, we derive the RG equations for a scale dependence of the averaged variables with a fixed time. There are three important fixed points including Einsteinde Sitter (EdS) universe. Setting the averaged variables at the horizon scale to the EdS values (because of inflation), we solve the RG equations toward the smaller scale to see the scale dependence of the observed cosmological parameters. We find that $\langle H\rangle$ increases toward smaller scale while $\Omega$ (三 $8 \pi G<\rho>/ 3<H>^{2}$ ) decreases, which may be favored by local observations of $H_{0}$ and $\Omega_{0}$. 